\title{
Influence of Imprecision on Credit Risk Assessment - Case Study
}

\section{Aleksandra WÓJCICKA-WÓJTOWICZ}

Poznań University of Economics and Business, Poznań, Poland; aleksandra.wojcicka-wojtowicz@ue.poznan.pl

\begin{abstract}
Credit risk is encountered in many situations that do not solely refer to loan granting process. In fact, the sophistication of nowadays global economy and the variety of utilised financial tools result in the existence of credit risk practically in any trade transaction. Therefore, credit risk assessment process is complex and is performed in many successive steps which cover multiple criteria. The importance of the problem on one hand calls for strictly analytical methods, however, on the other, also for a method which enables intuitive decision-making, imprecision and inaccurate linguistic ranks based on experts' personal experience. The paper presents the utility of Simple Additive Weighting method in a preceding phase of credit risk assessment. That step includes the preferences of experts on the significance of vital criteria burdened with imprecision, inaccurateness and linguistic labels. The presented illustrative example shows the importance of acknowledging experts' knowledge and perception of various linguistic, imprecise criteria. Therefore, the order scale is utilized.
\end{abstract}

Keywords: credit risk assessment; linguistic methods; SAW method; imprecision

JEL Classification: C02; C35; C38; G40

\section{Introduction}

Credit risk is understood as a probability of a borrower to default or to fail to fulfil their contractual obligations. An efficient management of credit risk improves banks' performance (Wójcicka-Wójtowicz 2018). Credit risk analysis is run in separate steps. Those steps usually cover a scrutinized analysis of borrowers' financial statements and are vital to a quantitative understanding of entity's condition. However, we must realise that the final decision is taken by a group of experts (higher level managers). Furthermore, each expert has their own preferences, scope of knowledge and life and business experience. Moreover, each of them expresses their preferences in real language using labels like "better/worse", "more/less", "higher/lower" etc. and still each of them can do it in a different manner or to a different extent. Consequently, we face a problem of an inaccurate and imprecise information. Yet, they can also use their experience not only to assess the current standing of the company but also to evaluate future projections of the borrower's business. Any existing collateral and future forecasts of the chosen industry are essential indicators in credit risk assessment. It is also not infrequent, that assessed features can be conflicting or excluding one another. The final decision can be consistent with the recommendation or it can reject the recommendation.

To utilise experts' opinions and preferences the weights (real numbers) of chosen groups of criteria are implemented. Moreover, each individual criterion within a given group is also given a weight. In this manner the experts' knowledge and professional experience is taken into consideration.

Furthermore, the main subject of the paper is the assessment of potential debtors, basing on chosen criteria, and the analysis is carried out by the means of multiple attributes decision-making approach based on Simple Additive Weighting (SAW) method which can deal with qualitative dimensions. The utilization of SAW provides experts with a support system allowing them to reach final decision based on linguistic, imprecise criteria. Also, Oriented Fuzzy SAW (OF-SAW) method (Piasecki and Roszkowska 2018) can be applied for solving the problem of evaluating the condition of the potential debtor. The proposed system integrates fuzzy set theory and SAW method to evaluate the available alternatives. 
The paper is organized as follows. Section 2 presents the overview of credit risk assessment methods. Section 3 outlines the basic facts on Ordered Fuzzy Numbers (OFN), general linguistic approach to the borrowers' evaluation and the implemented OF-SAW method. Section 4, being the main part of the research, shows the conducted case study implementing the weights for groups and individual criteria. The numerical example illustrates the procedure of the proposed SAW method and contributes into the understanding of the process of borrowers' evaluation. Finally, Section 5 presents the main findings of this research and possible future research directions.

\section{Credit Risk Assessment Techniques - Overview}

Banks can use various approaches to classify the borrowers. The methods, which are most widely used in credit risk assessment and the evaluation of borrowers, usually belong to a group of parametric methods such as linear discriminant analysis, regression analysis, credit scoring and non-parametric methods such as neural networks, expert systems or support vector machines, machine learning etc. All these methods have their limitations. Amongst those limitations we can enlist misclassification and indirect discrimination, variations from market to market, problems with accommodating changes, assuming a specific normality or homoscedasticity that are often violated in real world or model selection on trial and error process (Grace and Williams 2016).

Under Basel Capital Accord (1998) banks had to develop their Value-at-Risk methodology. In the scope of this approach that originated from standard and reduced models (so-called "new approach") we can find well-known models of rating agencies, consulting firms and other financial institutions, such as MKMV (originally proposed by Merton), CreditMetrics (introduced by RiskMetrics Group) and Credit Suisse Financial Product's CreditRisk+ or McKinsey's CreditPortfolioView, etc. These models, and their modifications, are constantly used by banks. However, it must be stressed that they are mostly successful in the USA due to calibration data used for their creation (more in Gordy 2000).

However, there is frequently a problem with applying those methods (no available data for nonquoted company on their market asset value) or with their ultimate efficiency due to their assumptions or strictly quantitative character of data which can lack the overall big picture.

Within the area of credit risk assessment and debtors' ratings there are also many modifications and extensions. A review on financial risk assessment (including credit and bankruptcy risks) can be found in Chen et al. (2016).

The new models and approaches appear as a result of the shortcomings of existing models. The significance of experts' knowledge and experience, as well as other qualitative factors in credit risk assessment and debtors' classification, are recognised as increasingly influential and helpful in decision-making process. In (Grace and Williams 2016) neural network and fuzzy logic systems for credit risk evaluation was developed and their performances were evaluated based on prediction accuracy metric. The conclusion was that despite comparable results, the fuzzy inference system could be easily understood by any user, however, the decisions made by the neural network system is not easily understood by the user, and in this case the user has no choice than to accept the output given by the neural network as the most appropriate output without any explicit reasoning. Also, in (Dadios and Solis 2012) a hybrid fuzzy logic and neural network algorithm (HFNN) to solve credit risk management problem is tested. It is shown that HFNN model can solve credit risk management problem and is capable of self- learning similar to the traditional neural network. It can also generate the rules behind the discrimination of each account subjected to it and in this manner, it behaves much like a traditional fuzzy logic system.

\section{Methodology}

\subsection{Ordered fuzzy numbers - basics}

Objects of any considerations may be given as elements of a predefined space $\mathbb{X}$. The basic tool for an imprecise classification of these elements is the notion of fuzzy sets introduced by Zadeh (1967). Any fuzzy set $\mathcal{A}$ is unambiguously determined by means of its membership function $\mu_{A} \in[0,1]^{\mathbb{X}}$, as follows 


$$
\mathcal{A}=\left\{\left(x, \mu_{A}(x)\right) ; x \in \mathbb{X}\right\}
$$

From the point-view of multi-valued logic (Łukasiewicz 1922/23), the value $\mu_{A}(x)$ is interpreted as the truth value of the sentence " $x \in \mathcal{A}$ ". By the symbol $\mathcal{F}(\mathbb{X})$ the family of all fuzzy sets in the space $\mathbb{X}$ is denoted.

The ordered FNs were intuitively introduced by Kosiński et al. (2002) as an extension of the FNs concept. Ordered FNs usefulness follows from the fact that it is interpreted as FNs with additional information about the location of the approximated number. A significant drawback of Kosiński's theory is that there exist such Kosiński's numbers which, in fact, are not FNs (Kosiński 2006). For this reason, the Kosiński's theory was revised by Piasecki (2018). If an ordered FN is determined with use of the revised definition, then it is called Oriented FN (OFN). The OFN definition fully corresponds to the intuitive Kosiński's definition of ordered FNs.

In this paper, the analysis is restricted to the case of Trapezoidal OFNs (TrOFN) defined as fuzzy subsets in the space $\mathbb{R}$ of all real numbers in the following way.

Definition 1: (Piasecki 2018) For any monotonic sequence $(a, b, c, d) \subset \mathbb{R}, \operatorname{TrOFN} \overleftrightarrow{\operatorname{Tr}}(a, b, c, d)=\overleftrightarrow{T}$ is the pair of the orientation $\overrightarrow{a, d}=(a, d)$ and a fuzzy subset $\mathcal{T} \in \mathcal{F}(\mathbb{R})$ determined explicitly by its membership functions $\mu_{T} \in[0,1]^{\mathbb{R}}$ as follows

$$
\mu_{T}(x)=\mu_{T r}(x \mid a, b, c, d)=\left\{\begin{array}{cc}
\mathbf{0}, \quad x \notin[\min \{a, d\}, \max \{a, d\}], \\
\frac{b-a}{b-a}, & x \in[\min \{a, b\}, \max \{a, b\}[, \\
\frac{x-d}{c-d}, & x \in[\min \{b, c\}, \max \{b, c\}],
\end{array}\right.
$$

The symbol $\mathbb{K}_{T r}$ denotes the space of all TrOFNs. Any TrOFN describes an imprecise number with additional information about the location of the approximated number. This information is given as orientation of OFN. If $a<d$ then $\operatorname{TrOFN} \overleftrightarrow{\operatorname{Tr}}(a, b, c, d)$ has the positive orientation $\overrightarrow{a, d}$. For any $z \in[b, c]$, the positively oriented $\operatorname{TrOFN} \overleftrightarrow{\operatorname{Tr}}(a, b, c, d)$ is a formal model of linguistic variable "about or slightly above $z^{\prime \prime}$. If $a>d$, then OFN $\overleftrightarrow{\operatorname{Tr}}(a, b, c, d)$ has the negative orientation $\overrightarrow{a, d}$. For any $z \in$ $[c, b]$, the negatively oriented $\operatorname{TrOFN} \overleftrightarrow{\operatorname{Tr}}(a, b, c, d)$ is a formal model of linguistic variable "about or slightly below $z$ ". Understanding the phrases "about or slightly above $z$ " and "about or slightly below $z^{\prime \prime}$ depends on the applied pragmatics of the natural language. If $a=d$, then $\operatorname{TrOFN} \overleftrightarrow{\operatorname{Tr}}(a, a, a, a)=$ $\llbracket a \rrbracket$ describes un-oriented real number $a \in \mathbb{R}$.

Kosiński has introduced the arithmetic operators of dot product $\odot$ for TrOFNs in a following way:

$$
\beta \odot \overleftrightarrow{\operatorname{Tr}}(a, b, c, d)=\overleftrightarrow{\operatorname{Tr}}(\beta \cdot a, \beta \cdot b, \beta \cdot c, \beta \cdot d)
$$

In (Piasecki 2018), the sum $\boxplus$ for TrOFNs is determined as follows

$$
\begin{gathered}
\overleftrightarrow{\operatorname{Tr}}(a, b, c, d) \boxplus \overleftrightarrow{\operatorname{Tr}}(p-a, q-b, r-c, s-d)= \\
\begin{cases}\overleftrightarrow{\operatorname{Tr}}(\min \{p, q\}, q, r, \max \{r, s\}) & (q<r) \vee(q=r \wedge p \leq s) \\
\overleftrightarrow{\operatorname{Tr}}(\max \{p, q\}, q, r, \min \{r, s\}) & (q>r) \vee(q=r \wedge p>s)\end{cases}
\end{gathered}
$$

Let us consider the pair $(\overleftrightarrow{\mathcal{K}}, \overleftrightarrow{\mathcal{L}}) \in \mathbb{K}_{T r}^{2}$ represented by the pair $\left(\mu_{K}, \mu_{L}\right) \in\left([0,1]^{\mathbb{R}}\right)^{2}$ of their membership functions. On the space $\mathbb{K}_{T r}$, the relation $\overleftrightarrow{\mathcal{K}} \cdot \widetilde{G E} \cdot \overleftrightarrow{\mathcal{L}}$, is introduced, which reads:

\section{"TrOFN $\overleftrightarrow{\mathcal{K}}$ is greater than or equal to TrOFN $\overleftrightarrow{\mathcal{L}}$."}

This relation is a fuzzy preorder $\widetilde{G E} \in \mathcal{F}\left(\mathbb{K}_{T r}^{2}\right)$ defined by its membership function $v_{G E} \in[0,1]^{\mathbb{K}_{T r}^{2}}$ (Piasecki 2019; Piasecki et al. 2019). From the point of view of the multivalued logic, the value $v_{G E}(\overleftrightarrow{\mathcal{K}}, \overleftrightarrow{\mathcal{L}})$ is considered as a truth-value of the sentence (5). In (Piasecki 2019), it is shown that for any pair $(\operatorname{Tr}(a, b, c, d), \operatorname{Tr}(e, f, g, h)) \in \mathbb{K}_{T r}^{2}$ we have 


$$
v_{G E}(\overleftrightarrow{\operatorname{Tr}}(a, b, c, d), \overleftrightarrow{\operatorname{Tr}}(e, f, g, h))=\left\{\begin{array}{cl}
0, & 0<\alpha-\gamma, \\
\frac{\alpha-\gamma}{\alpha+\delta-\beta-\gamma}, & \alpha-\gamma \leq 0<\beta-\delta, \\
1, & \beta-\delta \leq 0
\end{array}\right.
$$

where

$$
\begin{aligned}
& \alpha=\max \{a, d\} \\
& \beta=\max \{b, c\} \\
& \gamma=\min \{e, h\} \\
& \delta=\min \{f, g\}
\end{aligned}
$$

Therefore, for any pair $(\boldsymbol{T} \boldsymbol{T}(\boldsymbol{a}, \boldsymbol{b}, \boldsymbol{c}, \boldsymbol{d}), \llbracket \boldsymbol{e} \rrbracket) \in \mathbb{K}_{T r} \times \mathbb{R} \subset \mathbb{K}_{T r}^{2}$ we get

$$
v_{G E}(\overleftrightarrow{T r}(a, b, c, d), \llbracket e \rrbracket)=\left\{\begin{array}{cc}
\frac{\max \{a, d\}-e}{0}, \quad \max \{a, d\}<e, \\
\max \{a, d\}-\max \{b, c\} & \max \{a, d\} \geq e>\max \{b, c\}, \\
1, & 0 \leq c-f .
\end{array}\right.
$$

\subsection{Linguistic approach}

Credit risk managers and analysts realise that any credit granting decision is threatened by unpredictable default risk. To limit the potential losses, they evaluate borrowers in terms of many criteria.

Any borrower attributes can be evaluated by means of numerical values. By its very nature of things, each such assessment is an imprecise information. Therefore, when dealing with the imprecise information, the use of linguistic assessments may be more useful. Following (Herrera and HerreraViedma 2000), it can be said that an application of imprecise linguistic assessments for decision analysis is very beneficial because it introduces a more flexible framework which allows us to represent the information in a more direct and adequate way when it is difficult or impossible to express it precisely. However, by means of ranking systems, the qualitative concept can be translated into a quantitative one.

In the first step of any linguistic approach, the imprecision granularity should be determined, i.e., the cardinality of the linguistic term set used for showing the information. The imprecision granularity indicates the capacity of distinction that may be expressed. The knowledge value is increasing with the increase in granularity. The typical values of cardinality used in the linguistic models are odd ones, usually between 3 and 13. It is worth to note that the idea of granular computing goes from Zadeh (1997) who wrote "fuzzy information granulation underlies the remarkable human ability to make rational decisions in an environment of imprecision, partial knowledge, partial certainty and partial truth." Also, Yao (2004) pointed out that "the consideration of granularity is motivated by the practical needs for simplification, clarity, low cost, approximation ...". For review variety of application linguistic models in decision-making see for example (Herrera and Herrera-Viedma 2000).

In general (Herrera and Herrera-Viedma 2000), any linguistic value is characterized by means of a label with semantic value. The label is an expression belonging to a given linguistic term set. Finally, a mechanism of generating the linguistic descriptors is provided.

In credit risk assessment, all linguistic assessments are linked with Tentative Order Scale (TOS) given as a sequence

$$
\text { TOS }=\{\text { Bad , Average }, \text { Good }\}=\{C, B, A\}=\left\{V_{1}, V_{2}, V_{3}\right\}
$$

Any element of TOS is called a reference point and can be enlarged by intermediate values. For this purpose, the following orientation phrases can be used:

- "much below" described by the symbol " - -", 
- "below" described by the symbol " - ",

- "around" described by the symbol " ",

- "above" described by the symbol " +",

- "much above" described by the symbol " + +".

Any order label is determined as a composition of reference point and orientation phrases. The set of all order labels is called Extended Order Scale (EOS). In Table 1, TOS and EOS proposed for credit risk assessment are presented.

In information sciences, natural language word is considered as a linguistic variable defined as a fuzzy subset in the predefined space $\mathbb{X}$. Then, these linguistic variables may be transformed with the use of fuzzy set theory (Zadeh 1975). From decision making point view, the linguistic variable transformation methodologies are reviewed in (Herrera et al. 2009).

Let us assume that each reference point $V_{j}$ is represented by the number $j \in \mathbb{N}$. On the other side, the semantic meaning of any orientation phrase is imprecise. For this reason, any order label may be considered as imprecise approximation of its reference point. Thus, each order label from applied EOS should be represented in the real line $\mathbb{R}$ by FN (Chen and Hwang 1992). For convenience of future calculations, this representation can always be restricted to representation by trapezoidal FN. Moreover, the observation is made that orientation phrases determine the orientation of FN representing approximated reference point. Therefore, any order label can be represented by TrOFN. This approach is more faithful than representation of order labels by trapezoidal FN. On the other hand, an omission of information about order labels' orientation causes unbelievable assessment of borrowers (Piasecki et al. 2019 b). For these reasons, all order labels will be represented by TrOFNs. The family of all TrOFNs representing considered EOS will be called Numerical Order Scale (NOS). In credit risk assessment task, NOS is used. All applied order scales are presented in Table 1.

Table 1. Tentative Order Scale.

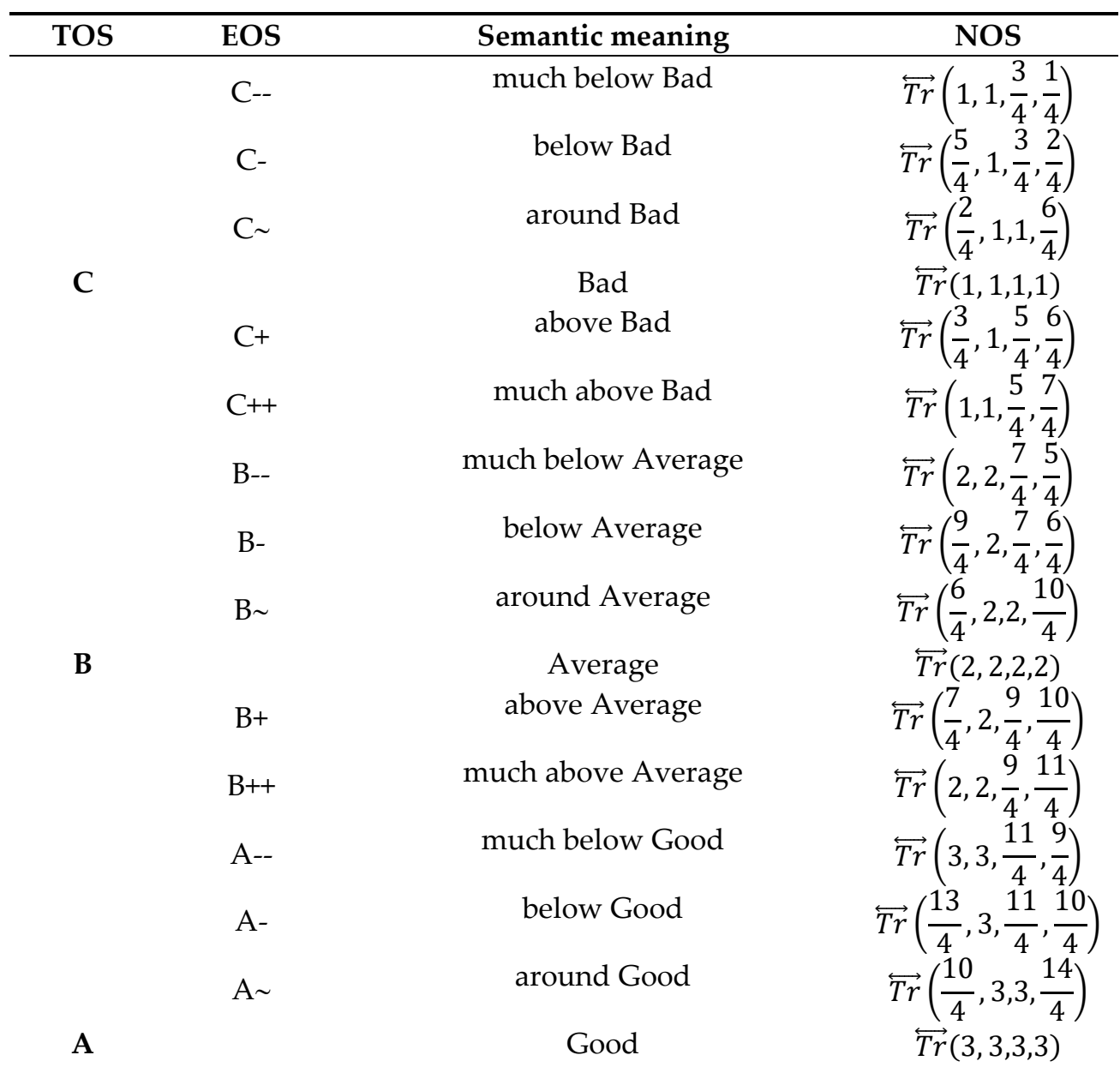




\begin{tabular}{ccc}
$\mathrm{A}+$ & above Good & $\overleftrightarrow{\operatorname{Tr}}\left(\frac{11}{4}, 3, \frac{13}{4}, \frac{14}{4}\right)$ \\
$\mathrm{A}++$ & much above Good & $\overleftrightarrow{\operatorname{Tr}}\left(3,3, \frac{13}{4}, \frac{15}{4}\right)$ \\
\hline
\end{tabular}

\subsection{Simple additive weighting method - overview}

To assess the potential debtor, we must have an evaluation template. This template distinguishes all borrower's attributes which are taken into consideration. The process of determining evaluation template is an important part of credit risk analysis, as well as constructing a scoring function, which is utilised in the pre-evaluation phase. Because borrowers are often characterized by several contradictory criteria, the multi-criteria techniques are useful for building borrower-scoring function. The most popular techniques used for multi-criteria evaluation is the Simple Additive Weighting (SAW) method (Mardani et al. 2015). The SAW method is a scoring method based on the concept of a weighted average of criterion ratings. In the considered task of a credit risk evaluation, the individual criterion ratings are expressed by TrOFNs. For this reason, SAW method linked with TrOFNs is needed. Such SAW method should be equipped with scoring function determined on the space $\mathbb{K}_{T r}^{n}=$ $\mathbb{K} \times \mathbb{K} \times \ldots \times \mathbb{K}$.

The SAW method is also called Simple Multi Attribute Rating Technique. In (Piasecki and Roszkowska 2018) Oriented Fuzzy SAW (OF-SAW) method is modified in a way that it is compatible with the revised theory of ordered FNs (Piasecki 2018). In this case, criterion ratings are given as TrOFNs. Below, the OF-SAW method is adapted to the needs of assessing a single borrower.

The intention is to evaluate a borrower characterized by attributes record $\mathcal{A} \in \mathbb{A}$ where $\mathbb{A}$ is an anticipated set of potential borrowers. For this case OF-SAW method can be described by the following procedure:

Step 1: Define a multi-criteria evaluation problem by criteria set $\mathbb{D}=\left\{\mathcal{C}_{1}, \mathcal{C}_{2}, \ldots, \mathcal{C}_{n}\right\}$.

Step 2: Determine the weight vector

$$
w=\left(w_{1}, w_{2}, \ldots, w_{n}\right) \in\left(\mathbb{R}_{0}^{+}\right)^{n}
$$

where

$$
w_{1}+w_{2}+\cdots+w_{n}=1 .
$$

and $w_{j}$ is the weight of the criterion $\mathcal{C}_{j}$ denoting the importance of this criterion in considered evaluation problem

Step 3: For each evaluation $\mathcal{C}_{j}(j=1,2, \ldots, n)$, determine its scope $Y_{j}$.

Step 4: Determine the evaluation template

$$
\mathbb{Y}=Y_{1} \times Y_{2} \times \ldots \times Y_{n} \supset \mathbb{A}
$$

Step 5: Define the NOS $\mathbb{O} \subset \mathbb{K}_{t r}$.

Step 6: Define the evaluation function $X: \mathbb{Y} \times \mathbb{D} \rightarrow \mathbb{O} \subset \mathbb{K}_{t r}$ in such way that the value $\chi\left(\mathcal{A}, \mathcal{C}_{j}\right) \in \mathbb{O}$ is equal to evaluation of attributes record $\mathcal{A}$ from the point-view of the criterion $\mathcal{C}_{j}$ $(j=1,2, \ldots, n)$.

Step 7: Determine the scoring function $\overleftrightarrow{S A W}: \mathbb{Y} \rightarrow \mathbb{K}_{T r}$ given for any $\mathcal{A} \in \mathbb{Y}$ by the identity

$$
\begin{aligned}
& \overleftrightarrow{S A W}(\mathcal{A})= \\
& =\left(w_{1} \odot \mathcal{X}\left(\mathcal{A}, \mathcal{C}_{1}\right)\right) \boxplus\left(w_{2} \odot \mathcal{X}\left(\mathcal{A}, \mathcal{C}_{2}\right)\right) \boxplus \ldots \boxplus\left(w_{n} \odot \mathcal{X}\left(\mathcal{A}, \mathcal{C}_{n}\right)\right)
\end{aligned}
$$

For a given evaluation template $\mathbb{Y}$, any classical scoring method of credit risk assessment can be presented as a pair $(f, L)$ (Mays 2001; Anderson 2007) where:

$f: \mathbb{Y} \rightarrow \mathbb{R}$ is a given scoring function,

$L \in \mathbb{R}$ is a predetermined level of acceptance of a credit/loan application.

Let us consider a credit application of a borrower characterized by attributes record $\mathcal{A} \in \mathbb{A}$. If the following condition is fulfilled

$$
f(\mathcal{A}) \geq L
$$


then the application is acceptable (Mays 2001; Anderson 2007).

In this section to assess the creditworthiness it is suggested to use a scoring function $\overleftrightarrow{S A W}: \mathbb{Y} \rightarrow \mathbb{K}_{T r}$. Therefore, it is also suggested to extend the inequality (16) into a following form

$$
\overleftrightarrow{S A W}(\mathcal{A}) . \widetilde{G E} . \llbracket L \rrbracket
$$

The fulfilment of the above inequality is tantamount to a sentence:

\section{Credit application based on attributes record $\mathcal{A}$ is acceptable}

Then the value $v_{G E}(\overleftrightarrow{S A W}(\mathcal{A}), \llbracket L \rrbracket)$ is truth-value of the sentence (19). For this reason, we interpret the value $v_{G E}(\overleftrightarrow{S A W}(\mathcal{A}), \llbracket L \rrbracket)$ as a degree in which the considered credit application is acceptable. Therefore, the value

$$
\operatorname{accept}(\mathcal{A}, L)=v_{G E}(\overleftrightarrow{S A W}(\mathcal{A}), \llbracket L \rrbracket)
$$

will be called the acceptance degree (acceptance level). This value can be a significant premise for the credit committee to take a final decision to grant the funding.

\section{Numerical Example - Case Study}

The data was collected from two experts in the banking field who are active members of a credit assessment committee with a long business experience in that field (the personal data of experts and any data concerning the Bank as well as any enterprise and decision-making actions involved in the process, are subject to confidentiality). The research was conducted in the following steps:

Step 1. Prepare an appropriate assessment form (template).

During the process of template preparation, experts were to indicate a criteria which they considered vital for credit risk assessment of potential borrower. The lists of chosen criteria were compared. Eventually, 16 criteria, which appeared in case of both experts, were chosen and applied. Those criteria are qualitative and positive for selecting a good potential debtor amongst the analysed ones and ranking them. The introduced method is used in a case study. The chosen criteria are presented in Table 2.

Step 2. Incorporate weights of the criteria

In the research basing on the experts' professional knowledge the criteria were divided into 5 groups (see Table 2). Each group was given a ranking grade (a rank) of a real number ranging from 1 to 5 where 1 meant "the least important group" and 5 meant "the most important group". The weights of each group were calculated as a quotient of the given rank (respectively 1,2,3,4 or 5) and the sum of the ranks (15). This way the least important group had the weight $1 / 15$ and the most important group was given the weight of $5 / 15$. Also, each individual criterion within a given group was attributed a weight depending on their significance. The ranks (respectively $\{1,2\},\{1,2,3\}$ or $\{1,2,3,4\}$ - depending on the group) show the importance of the individual criterion in its group. Accordingly, 1 meant "the least important", while the highest value indicated "the most important" criterion. The weights of each criterion in a given group were calculated as a quotient of the given rank (respectively 1,2,3 or 4) and the sum of the ranks (10). This way, the least important criterion in group with ranks $\{1,2,3,4\}$ had the attributed weight of $1 / 10$ and the most important criterion in that group was given $4 / 10$, In other groups it was respectively $1 / 6$ and $3 / 6$ for $\{1,2,3\}$ and $1 / 3$ and $2 / 3$ for $\{1,2\}$.

All incorporated weights (for groups and criteria) are presented in Table 2. Groups of criteria are presented from the most important to the least important. 
Table 2. Weights for groups and criteria.

\begin{tabular}{|c|c|c|}
\hline $\begin{array}{l}\text { rank of the } \\
\text { group (1-5) } \\
\text { / weight of } \\
\text { the group }\end{array}$ & criteria / (weight in the group) & $\begin{array}{l}\text { weights of an } \\
\text { individual criterion } \\
\text { (within the groups) }\end{array}$ \\
\hline \multirow{4}{*}{$5 / 0,333$} & risk of the market / $(0,4)$ & 0,133 \\
\hline & risk of the trade $/(0,3)$ & 0,1 \\
\hline & risk of the supplier / $(0,1)$ & 0,033 \\
\hline & risk of the customer / $(0,2)$ & 0,067 \\
\hline \multirow{3}{*}{$4 / 0,267$} & diversification of the product / $(0,333)$ & 0,089 \\
\hline & diversification of the sale markets $(0,5)$ & 0,133 \\
\hline & diversification of the supply market $(0,167)$ & 0,044 \\
\hline \multirow{3}{*}{$3 / 0,2$} & prospects of business / $(0,5)$ & 0,1 \\
\hline & quality of suppliers / $(0,167)$ & 0,033 \\
\hline & quality of customers / $(0,333)$ & 0,067 \\
\hline \multirow{4}{*}{$2 / 0,133$} & clean criminal record of the Board members / $(0,1)$ & 0,013 \\
\hline & clean criminal record of a chairperson / $(0,2)$ & 0,026 \\
\hline & experience of the Board members / $(0,3)$ & 0,04 \\
\hline & experience of a chairperson / $(0,4)$ & 0,053 \\
\hline \multirow{2}{*}{$1 / 0,067$} & operations range - Poland / $(0,667)$ & 0,044 \\
\hline & operations range - abroad / $(0,333)$ & 0,022 \\
\hline
\end{tabular}

Step 3. Set the acceptance level.

The acceptance level was established in "a middle point between reference points 'Average' and 'Good' ". The experts were given that information.

Step 4. Experts fill in the form.

The experts express their individual, professional opinion on the specific criterion in relation to an analyzed enterprise by attributing that criterion to a single rank of EOS.

Step 5. Transform the experts' evaluations into NOS.

The evaluations given by each expert were transformed into NOS.

As experts can have different preferences and different professional experience, the obtained results reflect the differences in perception of the importance of qualitative features in case of assessing the same entity. Therefore, as a final assessment, the mean SAW value, representing common opinion of both experts, was calculated (Table 3 ).

Table 3. Values of scoring SAW function.

\begin{tabular}{cccc}
\hline & & Evaluations & \\
\hline Enterprise & SAW by Expert 1 & SAW by Expert 2 & Mean SAW \\
A & $\overleftrightarrow{\operatorname{Tr}}\left(\frac{68}{32}, \frac{73}{32}, \frac{77}{32}, \frac{85}{32}\right)$ & $\overleftrightarrow{\operatorname{Tr}}\left(\frac{130}{64}, \frac{130}{64}, \frac{133}{64}, \frac{147}{64}\right)$ & $\overleftrightarrow{\operatorname{Tr}}\left(\frac{135}{128}, \frac{138}{128}, \frac{144}{128}, \frac{157}{64}\right)$ \\
B & $\overleftrightarrow{\operatorname{Tr}}\left(\frac{125}{64}, \frac{133}{64}, \frac{140}{64}, \frac{151}{64}\right)$ & $\overleftrightarrow{\operatorname{Tr}}\left(\frac{149}{64}, \frac{152}{64}, \frac{157}{64}, \frac{176}{64}\right)$ & $\overleftrightarrow{\operatorname{Tr}}\left(\frac{267}{128}, \frac{280}{128}, \frac{304}{128}, \frac{329}{128}\right)$ \\
\hline
\end{tabular}

In the next step, for each assessed value of SAW scoring function the acceptance degree values are calculated (20). Due to the fact that the acceptance level is represented by "a middle point between reference points 'Average' and 'Good'", it is assumed that the acceptance level is given as

$$
L=\frac{1}{2} \cdot(2+3)=\frac{5}{2}
$$

Here, we utilize the relationship (11). All values acquired in this manner are presented in Table 4. 
Table 4. Acceptance level (by each expert and Mean SAW).

\begin{tabular}{cccc}
\hline & \multicolumn{3}{c}{ Acceptance degree } \\
\hline Enterprise A & by Expert 1 & by Expert 2 & of Mean SAW \\
Enterprise B & 0.385 & 0.125 & 0.000 \\
\hline
\end{tabular}

The values presented in Table 4 allow to formulate the following findings:

- $\quad$ credit application of Enterprise A is accepted at medium level by Expert 1;

- credit application of Enterprise A is accepted at a low level (weak acceptance) by Expert 2;

- credit application of Enterprise A is not accepted by experts' team;

- credit application of Enterprise B is accepted at a low level (weak acceptance) by Expert 1;

- credit application of Enterprise B is strongly accepted by Expert 2;

- credit application of Enterprise B is accepted at medium level by experts' team.

The final decision of granting the credit (loan) is up to the credit committee. The committee can take into consideration the opinions presented above. The fact, that experts' knowledge is often underappreciated, yet, it can significantly influence final decision must be, eventually, noticed and methods of behavioral finance combined with a "soft" approach such as OFNs and linguistic techniques must be developed and implemented.

\section{Conclusions}

Credit risk assessment is a complex process. Credit risk managers try to mitigate the effects of the credit risk threat by utilising various techniques of borrowers' assessment. Those techniques influence the final decision of granting the funding or rejecting the application. In decision-making process there is a number of stages which consist of numerous criteria. Also, ordering the decision alternatives is an important part of the whole decision-making analysis which takes place before making a final decision. The nature of the problem enables intuitive decision-making, imprecision and inaccurate linguistic ranks based on experts' personal experience.

The calculations, conducted in a numerical example presented in the paper, show the utility of SAW method in case of a credit risk assessment and the order scale is described by oriented fuzzy numbers (OFN).

The presentation and estimation of the acceptance level should be a subject of further research.

Acknowledgments: I would like to express my deepest gratitude to Professor Krzysztof Piasecki (Poznań University of Economics and Business) for all his invaluable remarks and insight. I would like to extend my sincere thanks to Anna Łyczkowska-Hanćkowiak (WSB) for the support.

\section{References}

Chen Shu-Jen, and Ching-Lai Hwang. 1992. Fuzzy Multiple Attribute Decision Making Methods. In Economics and Mathematical Systems Fuzzy Multiple Attribute Decision Making. Berlin: Springer-Verlag. 289-486., doi:10.1007/978-3-642-46768-4_5.

Dadios P. Elmer. 2012. Fuzzy-Neuro Model for Intelligent Credit Risk Management. Intelligent Information Management: 02(25), 251-260. https://doi.org/10.4236/iim.2012.225036.

Dubois Didier, and Prade Henri. 1979. Fuzzy real algebra: Some results. Fuzzy Sets and Systems: 2(4), 327-348. doi: 10.1016/0165-0114(79)90005-8

Grace M. Asogbon, and Williams S. Oluwarotimi. 2016. Comparative Analysis of Neural Network and Fuzzy Logic Techniques in Credit Risk Evaluation. International Journal of Intelligent Information Technologies: 12(1), 47-62. doi: 10.4018/ijiit.2016010103

Herrera Francisco, and Herrera-Viedma Enrique. 2000. Linguistic decision analysis: steps for solving decision problems under linguistic information. Fuzzy Sets and Systems: 115(1), 67-82. doi: 10.1016/s01650114(99)00024-x

Herrera Francisco, Alonso Salazar, Chiclana Francisco, and Herrera-Viedma, Enrique. 2009. Computing with words in decision making: foundations, trends and prospects. Fuzzy Optimization and Decision Making: 8(4), 337-364. doi: 10.1007/s10700-009-9065-2 
Kanapickiene Rasa, and Spicas Renatas. 2019. Credit Risk Assessment Model for Small and Micro-Enterprises: The Case of Lithuania. Risks: 7(2), 67. doi: 10.3390/risks7020067

Kosiński Witold, Prokopowicz Piotr, and Ślęzak Dominik. 2002 Drawback of fuzzy arithmetics - new intuitions and propositions. Methods of Artificial Intelligence Burczyński T, Cholewa W, Moczulski W (eds), Gliwice. Poland:231-237

Kosiński Witold. 2006 On fuzzy number calculus. Int. J. Appl. Math. Comput. Sci.: 16(1):51-57

Łukasiewicz Jan. 1922/23, Interpretacja liczbowa teorii zdań, Ruch Filozoficzny: 7, 92-93. Translated as 'A numerical interpretation of the theory of propositions' In Jan Łukasiewicz - Selected Works, Borkowski, L. (eds.), NorthHolland, Amsterdam, Polish Scientific Publishers: Warszawa, Poland, 1970

Mardani Abbas, Jusoh Ahmad, and Zavadskas K. Edmundas. 2015. Fuzzy multiple criteria decision-making techniques and applications - Two decades review from 1994 to 2014. Expert Systems with Applications: 42(8), 4126-4148. doi: 10.1016/j.eswa.2015.01.003

Piasecki Krzystof. 2019. Relation "Greater than or Equal to" between Ordered Fuzzy Numbers. Applied System Innovation: 2(3), 26. doi: 10.3390/asi2030026

Piasecki Krzysztof, and Roszkowska Ewa. 2018. On Application of Ordered Fuzzy Numbers in Ranking Linguistically Evaluated Negotiation Offers. Advances in Fuzzy Systems: 1-12. doi: 10.1155/2018/1569860

Piasecki Krzysztof. 2018. Revision of the Kosiński's Theory of Ordered Fuzzy Numbers. Axioms: 7(1), 16. doi: 10.3390/axioms7010016

Piasecki Krzysztof., Łyczkowska-Hanćkowiak Anna, Wójcicka-Wojtowicz Aleksandra. 2019. The relation "greater than or equal to" for trapezoidal ordered fuzzy numbers, 37th International Conference on Mathematical Methods in Economics 2019, Conference Proceedings, Houda M, Remeš R, (eds), University of South Bohemia in České Budějovice, Faculty of Economics, České Budějovice, Czech Republic: 61-66

Wójcicka-Wójtowicz Aleksandra. 2018. Credit Risk Management in Finance. A Review of Various Approaches. Operations Research and Decisions: 28(4), 99-106. htttps://doi: 10,5277/ord180407

Yao Yiyu. 2004 Granular computing, Computer Science: 31, 1-5

Zadeh A. Lotfi. 1965. Fuzzy Sets. Information and Control: 8(3), 338-353. doi: 10.21236/ad0608981

Zadeh A. Lotfi. 1975. The concept of a linguistic variable and its application to approximate reasoningI. Information Sciences: 8(3), 199-249. doi: 10.1016/0020-0255(75)90036-5s 